\title{
Wireless Tissue Palpation: Proof of Concept for a Single Degree of Freedom
}

\author{
Marco Beccani, Christian Di Natali, Mark E. Rentschler, Pietro Valdastri
}

\begin{abstract}
Palpating tissues and organs to identify hidden tumors or to detect buried vessels is not a viable option in laparoscopic surgery due to lack of force feedback. So far, research toward restoring tactile and kinesthetic sensations in minimally invasive surgery has focused on the distal sensing element or on the proximal rendering of haptic cues. In this work we present a pilot study to assess the feasibility of wireless tissue palpation, where a magnetic device is deployed through a standard surgical trocar and operated to perform tissue palpation without requiring a dedicated entry port. The setup consists of a wireless intra-body device and an external robotic manipulator holding a load cell and a permanent magnet. Embedded in the wireless cylindrical device $(12.7 \mathrm{~mm}$ in diameter and $27.5 \mathrm{~mm}$ in height) is a sensing module, a wireless microcontroller, a battery and a permanent magnet. This preliminary study assessed the precision in reconstructing the indentation depth based on magnetic field measurements at the wireless device (i.e., $0.1 \mathrm{~mm}$ accuracy). Experimental trials demonstrated the effectiveness of wireless vertical indentation in detecting the elastic modulus of three different silicone tissue simulators (elastic modulus ranging from $50 \mathrm{kPa}$ to $93 \mathrm{kPa}$ ), showing a maximum relative error below $3 \%$. Finally, wireless palpation was used to identify differences in tissue stiffness from a lump embedded into a porcine liver. The reported results have the potential to open a new paradigm in the field of palpation devices, where direct physical connection across the abdominal wall is no longer required.
\end{abstract}

\section{INTRODUCTION}

$\mathbf{T}$ ODAY minimally invasive surgery (MIS) is a popular and widely accepted clinical practice, with more than two million procedures performed in the United States annually [?]. Robotic MIS is also increasingly common with more than 2,000 of Intuitive Surgical's da Vinci platforms installed worldwide [1]. Despite the clear benefits in terms of patient recovery time, pain and scarring after the surgery, both MIS and robotic MIS prevent the surgeon from directly manipulating tissues and organs as is done in open surgery [2]. In particular, laparoscopic instruments used in MIS severely compromise any haptic cues because of friction against the surgical port (i.e., trocar) and the fulcrum effect at the insertion point [3]. The situation gets even worse when considering commercially available robotic MIS platforms, where zero haptic force feedback is available, since the surgical instruments are teleoperated from a remote console. Therefore, in MIS and robotic MIS the surgeon has minimal, or no, chance to leverage tactile and kinesthetic sensations in preventing

M. Beccani, C. Di Natali, P. Valdastri are with the STORM Lab, Department of Mechanical Engineering, Vanderbilt University, Nashville, TN 37235-1592. Email: p.valdastri@vanderbilt.edu

M. E. Rentschler is with the Department of Mechanical Engineering, University of Colorado, Boulder, CO 80303.

Email: mark.rentschler@colorado.edu

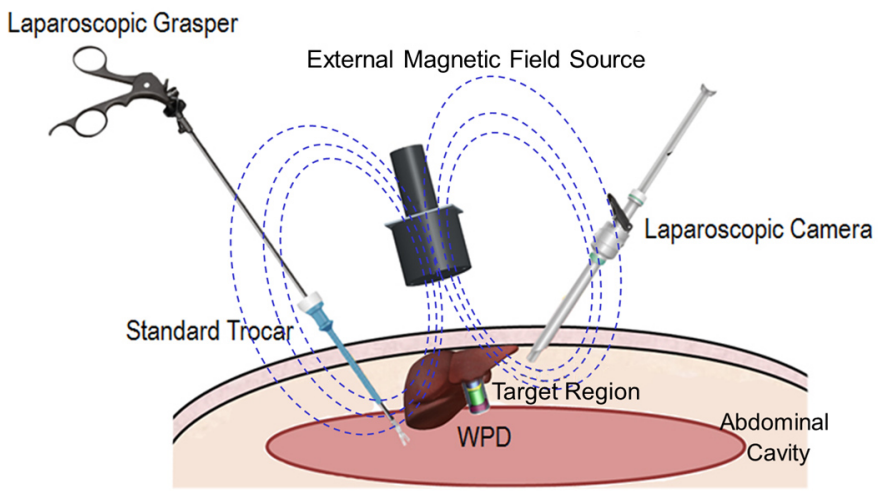

Fig. 1. Principle of operation for wireless tissue palpation using a wireless palpation device (WPD).

accidental tissue damage or to explore non-visible tissue and organ features by palpation.

Restoring tactile and kinesthetic sensations in MIS and robotic MIS has been an active research topic for more than two decades [4], [5], with one of the first systems used in a human dating back 1994 [6]. A relevant number of MIS instruments with force and/or tactile sensors have been developed to acquire in vivo data for tissue modeling and simulation [7]-[10], to improve the outcomes of the surgical procedure - preventing excessive forces from being applied to the tissues [3], [11]-[14], or to explore tissues and organs by palpation [2], [15]-[17]. This last functionality is particularly relevant whenever location and boundaries of a hidden tumor must be located - registration with pre-operative imaging is not effective for soft tissues [2], [16], or to identify buried structures (e.g., nerves or blood vessels) that must be avoided during the surgical procedure. MIS devices for tissue palpation developed to date exploit different approaches, such as grasping [5], [9], [12], rolling indentation [2], or vertical indentation [7], [8], [10], [15], [16].

However, the fact that no commercial MIS instruments with embedded force and/or tactile sensors have been adopted clinically so far [18] illustrates that there exists a significant roadblock preventing the translation of potentially transformative prototypes into clinical practice. Focusing on MIS palpation devices, a possible barrier may be that surgeons are not yet willing to devote a surgical port to an instrument whose sole purpose is to palpate tissues. Therefore, providing a tissue indenter that does not require port space may overcome this potential adoption barrier. 
In this spirit, we propose a wireless approach to tissue indentation, where a magnetic palpation device can be deployed through a standard trocar and operated without requiring a dedicated entry port. In other instances, the patient could swallow a wireless capsule that is used to remotely palpate organs through the gastrointestinal tract. Given the novelty of the approach, this work aims to demonstrate the feasibility of wireless indention for a single direction, leaving the extension to multiple degrees of freedom to future studies.

\section{MATERIALS}

\section{A. Principle of Operation}

Referring to Fig. 1, the approach we propose takes advantage of an external magnetic field source and an intrabody wireless palpation device (WPD) - embedded with a miniature permanent magnet and wireless electronics. The WPD can be introduced into the abdominal cavity through a standard trocar and positioned on the target by a laparoscopic grasper. Then, tissue indentation can be obtained by properly modulating the gradient of the external magnetic field. In order to generate kinesthetic data, the indentation depth and the pressure applied on the tissue must be known at any given time. In this pilot study, we restricted the investigation to a single degree of freedom (i.e., vertical indentation) as a first step toward proving the feasibility of the proposed approach.

A permanent magnet mounted at the end effector of a robotic manipulator was adopted as external magnetic field source. Considering the two magnets (i.e., the one inside the WPD and the one at the external manipulator) oriented as in Fig. 2, we studied the indentation of a tissue sample along the vertical direction by cyclically translating the external magnet along the $\mathrm{Z}$ axis. Neglecting gravity and assuming a pure vertical motion for the WPD, the pressure exerted on the tissue is provided by the ratio of the intermagnetic force along the $\mathrm{Z}$ axis, $F_{z}$, and the area of the WPD face in contact with the tissue. At the equilibrium, the intensity of $F_{z}$ can be measured by placing a load cell in between the external permanent magnet and the end effector of the manipulator, as suggested in [19]. For vertical indentation as represented in Fig. 2, gravitational force acting on the WPD can be considered as a preload on the tissue and factored out as an offset in the indentation trial. For any other configuration, an accelerometer can be embedded in the WPD to provide the inclination, thus allowing to quantify the exact contribution of the gravity force, should this vary during indentation. In this work, the inertial sensor is primarily used to verify the assumption of pure vertical motion for the WPD.

The indentation depth $\delta(t)$ can be evaluated by measuring the $\mathrm{Z}$ component of the magnetic field at the WPD. In particular, referring to Fig. 2 and focusing on the tissue loading phase, it is possible to express the distance between the external magnet and the internal magnet at the generic instant $t$ as:

$$
d(t)=d\left(t_{0}\right)-\delta(t)-d_{R}\left(t_{0}, t\right)
$$

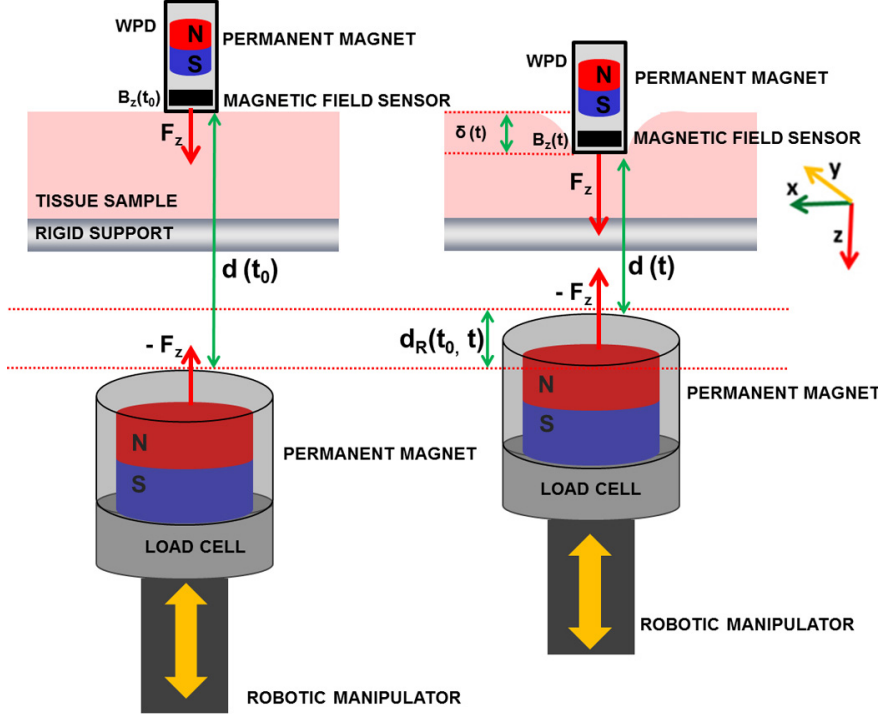

Fig. 2. Schematic diagram for wireless vertical indentation at the initial time $t_{0}$ (left) and at a generic time $t$ (right) during the loading phase.

where $d_{R}\left(t_{0}, t\right)$ is the vertical distance traveled by the robotic manipulator since the beginning of the loading phase occurred in $t_{0}$. Since the motion of the external magnet is limited to the $\mathrm{Z}$ axis and the WPD is aligned on that same direction in virtue of magnetic coupling, we can assume that the $\mathrm{Z}$ component of the magnetic field at the WPD, $B_{z}(t)$, is an univocal function of $d(t)[20]$ :

$$
B_{z}(t)=\Phi[d(t)]
$$

that can be numerically quantified through experimental calibration. Therefore, the indentation depth $\delta(t)$ can be expressed by merging Eq. 2 with Eq. 1 and rearranging the terms as:

$$
\delta(t)=\Phi\left[B_{z}\left(t_{0}\right)\right]^{-1}-\Phi\left[B_{z}(t)\right]^{-1}-d_{R}\left(t_{0}, t\right) .
$$

Since the value of $d_{R}\left(t_{0}, t\right)$ is available from the robotic manipulator encoders and $B_{z}(t)$ can be measured by placing a Hall effect sensor in the WPD, the total indentation depth can be computed at any given time during the loading phase. The same mathematical formulation applies - mutatis mutandis to the tissue unloading phase.

A relevant assumption for the proposed approach consists of considering all the tissue deformation occurring at the interface with the WPD. This holds true for the schematization represented in Fig. 2 - where the tissue being tested is laying on a rigid support. However, it may not be valid during in vivo conditions, where a stiffer organ may lay on a softer tissue. This approximation is, however, well accepted in the field of in vivo tissue indentation, as long as the indentation depth is relevantly smaller (approximately $10-15 \%$ of the total thickness) than the organ under test.

\section{B. Experimental Platform Overview}

The experimental platform used to assess wireless tissue palpation for a single degree of freedom is represented in Fig. 


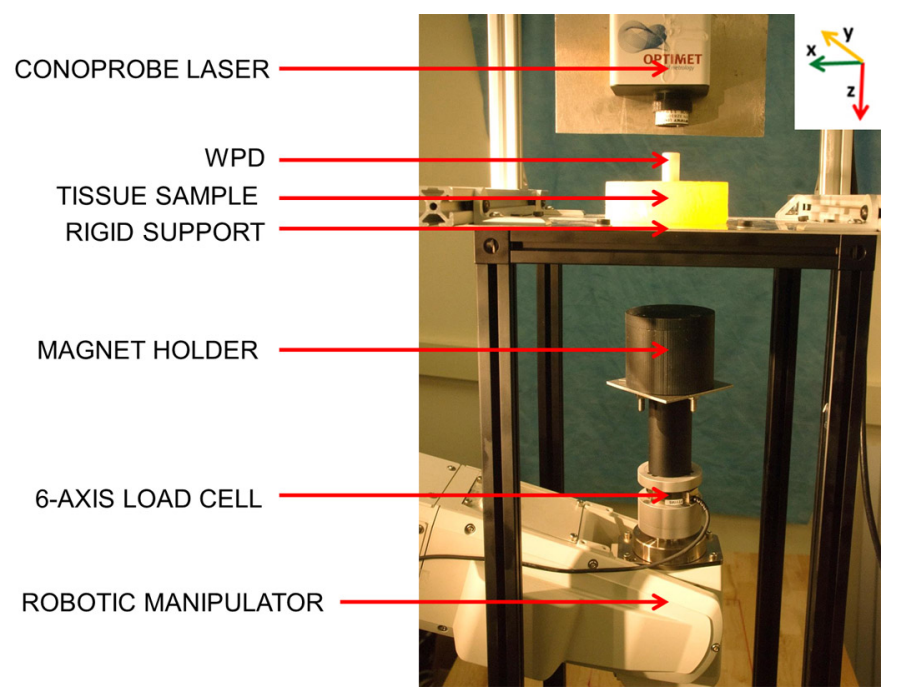

Fig. 3. Robotic platform for wireless vertical indentation. The four edges of the rigid support lay on a black supporting frame. The conoprobe laser is used for indentation depth validation.

3. It mainly consists of the WPD, the robotic manipulator, and the tissue being tested.

The WPD cylindrical shell is embedded with a permanent magnet, a sensing module, a wireless microcontroller, and a battery (Fig. 4). We selected an off-the-shelf cylindrical $\mathrm{NdFeB}$ permanent magnet (K\&J Magnetics, Inc., USA), 11 $\mathrm{mm}$ in diameter and $11 \mathrm{~mm}$ in height, with $\mathrm{N} 52$ axial magnetization (magnetic remanence of $1.48 \mathrm{~T}$ ). The sensing module consisted of a Hall effect sensor (CYP15A, Chen Yang Technologies $\mathrm{GmbH} \&$ Co. KG, Germany) to measure $B_{z}$, and a triaxial accelerometer (LIS331AL, STMicroelectronics, Switzerland) - to verify that the WPD motion during indentation was limited to the $\mathrm{Z}$ direction.

An analog signal conditioning stage connected to the Hall effect sensor output allowed for canceling out the offset due to the onboard permanent magnet (i.e., $100 \mathrm{mT}$ ), for applying a low-pass filter (cut-off frequency of $30 \mathrm{~Hz}$ ), and for amplifying the magnetic field signal (x29), resulting in a resolution of $0.32 \mathrm{mT}$ and a sensing range of $\pm 130 \mathrm{mT}$. An analog to digital converter (ADC) (ADS8320, Texas Instrument, USA) was used to acquire this voltage with a sampling rate of $1 \mathrm{kHz}$ and a resolution of 16 bits. The result of the conversion was then transmitted through a serial synchronous interface to the wireless microcontroller (CC2530, Texas Instruments, USA). The signals generated by the accelerometer - that did not required a 16-bit resolution for the sake of their purpose - were acquired directly by the microcontroller through its embedded 12-bit ADC at $100 \mathrm{~Hz}$. Accuracy results after digitalization were $0.35 \mathrm{mT}$ for the Hall effect sensor and 1.4 degree for the accelerometer used as inclinometer. Real-time clock timestamps were associated with each single measurement to enable synchronization with signals acquired by the external platform. The data were transmitted over a $2.4 \mathrm{GHz}$ carrier frequency to a receiving unit located in the same room and connected to a personal computer, where data were unbundled,
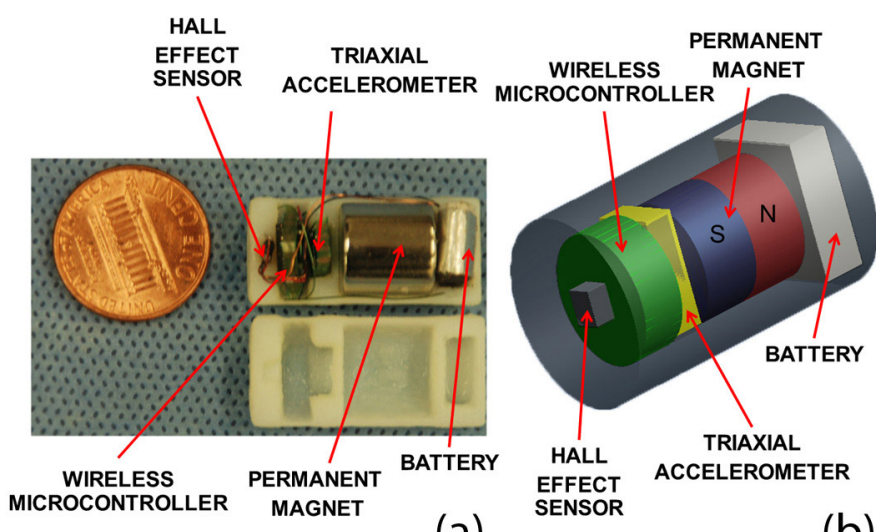

(a)

(b)

Fig. 4. Picture (a) and schematic view (b) of the wireless palpation device.

displayed, and stored. The use of a $2.4 \mathrm{GHz}$ carrier frequency was demonstrated to be effective in transmitting data through living tissues in [21], [22]. The wireless microcontroller was integrated into a custom-made $9.8 \mathrm{~mm}$ diameter printed circuit board, together with radiofrequency components. A digital switch driven by the microcontroller was placed between the battery and the sensing circuitry, to save battery power when measurements were not required.

A $15 \mathrm{mAh}, 3.7 \mathrm{~V}$ rechargeable LiPo battery (030815, Shenzhen Hondark Electronics Co., Ltd., China) was used as the onboard power supply. The battery layout (originally $8 \mathrm{~mm}$ $\times 15 \mathrm{~mm} \times 3 \mathrm{~mm}$ ) was reduced to fit the cylindrical shell. Considering that data acquisition and transmission requires an average of $33 \mathrm{~mA}$, battery lifetime was almost 30 minutes. Operational lifetime can easily be extended to fit application requirements by maintaining the WPD in sleep mode (average current consumption of $1.5 \mu \mathrm{A}$ ) and waking up the system by remote triggering whenever a palpation task is going to be performed.

As represented in Fig. 4, all the components were integrated inside a cylindrical plastic shell fabricated by rapid prototyping (OBJECT 30, Object Geometries Ltd, USA). Thanks to its small size $(12.7 \mathrm{~mm}$ in diameter and $27.5 \mathrm{~mm}$ in height), the WPD can be introduced through a 12-mm surgical trocar (e.g., the 5-12 Vesaport Plus, Covidien, USA has an inner diameter of $13 \mathrm{~mm}$ ). An axial-symmetric design was pursued in order to keep the WPD center of mass along its main axis, thus guaranteeing an uniform pressure on the tissue. Considering vertical indentation, the WPD surface area in contact with the tissue was $113 \mathrm{~mm}^{2}$, while the total weight was $117 \mathrm{mN}$ (i.e., WPD mass was $12 \mathrm{~g}$ ). It is worth mentioning that a tether can be connected to the WPD, should the surgeon feel the need for a fast retrieval of the palpation device in case of failure.

Concerning the external part of the platform - represented in Fig. 3 - an off-the-shelf cylindrical $\mathrm{NdFeB}$ permanent magnet (50 $\mathrm{mm}$ in diameter and $50 \mathrm{~mm}$ in height, weight $772 \mathrm{~g}$ ), with N52 axial magnetization (magnetic remanence of 1.48 T), was adopted. Considering an average thickness of the abdominal wall upon insufflation of $30 \mathrm{~mm}$ [23], this magnet 
was selected on the basis of numerical analysis [24] to operate at a distance along $Z$ ranging from $35 \mathrm{~mm}$ to $75 \mathrm{~mm}$ away from the WPD. In this region, the simulated absolute values of the field gradient range from $3.75 \mathrm{~T} / \mathrm{m}$ to $0.6 \mathrm{~T} / \mathrm{m}$, respectively. Considering the features of the magnet embedded in the WPD, the expected intermagnetic force spans from $4.7 \mathrm{~N}$ to 0.75 N. Should the required working distance be increased due to specific patient constraints (e.g., larger body mass index), an external magnet with different features can be selected by running numerical simulations again.

The magnet was embedded in a plastic holder connected to a 6-axis load cell (MINI45, Ati Indutrial Automation, Inc., USA), having a resolution of $65 \mathrm{mN}$ for the $\mathrm{Z}$ component of the force. The magnet-load cell assembly was mounted at the end effector of a six degrees of freedom industrial robot (RV6SDL, Mitsubishi Corp., Japan), presenting a motion resolution of $1 \mu \mathrm{m}$ along the $\mathrm{Z}$ direction. It is worth mentioning that the holder was designed so to space the magnet enough from the load cell and the manipulator to prevent electromagnetic interferences. Data from the load cell were acquired by a dedicated acquisition board (NI-PCI 6224, National Instruments, USA) at a sampling frequency of 10 $\mathrm{kHz}$, and merged with the manipulator position and the signals coming from the WPD.

The tissue sample being tested - silicone samples (MF Liquid Plastic, MF Manufacturing, USA) with different stiffnesses or porcine liver, depending on the trial - was placed on a $2 \mathrm{~mm}$ thick rigid support, as represented in Fig. 3.

Finally, the algorithm described by Eq. 3 was implemented in Matlab (Mathworks, USA) upon experimental calibration. In particular, the numerical function $\Phi^{-1}$ was evaluated by placing the WPD directly on the rigid support and by recording $B_{z}(t)$ while moving the external magnet at a constant speed (i.e., $3.12 \mathrm{~mm} / \mathrm{s}$ ) from a starting position $75 \mathrm{~mm}$ away from the rigid support along the $\mathrm{Z}$ axis (i.e., $d_{R}$ varying from 0 $\mathrm{mm}$ to $75 \mathrm{~mm}$, where for $d_{R}=75 \mathrm{~mm}$ the top part of the holder was almost in contact with the lower side of the rigid support). This measurement was performed for five loadingunloading cycles, and the values were averaged. Given the exponential decay of the magnetic field with distance, a fifthorder polynomial function was used to fit $\Phi^{-1}$, thus obtaining:

$$
d(t)=\Phi^{-1}\left[B_{z}(t)\right]=\sum_{i=0}^{5} a_{i} \cdot B_{z}(t)^{i}
$$

with $a_{0}=185.6 \mathrm{~mm}, a_{1}=-6.95 \cdot 10^{3} \mathrm{~mm} / \mathrm{T}, a_{2}=1.57 \cdot 10^{4}$ $\mathrm{mm} / \mathrm{T}^{2}, a_{3}=-2 \cdot 10^{7} \mathrm{~mm} / \mathrm{T}^{3}, a_{4}=1.31 \cdot 10^{7} \mathrm{~mm} / \mathrm{T}^{4}, a_{5}=$ $-3.51 \cdot 10^{7} \mathrm{~mm} / \mathrm{T}^{5}$. The square of the correlation coefficient for the proposed fitting was $R^{2}=0.99998$.

Since we are proposing a novel method to quantify the elastic modulus of the material being tested, it is interesting to analyze in detail the error we should expect. Assuming vertical indentation, the error in measuring the stress straightforwardly depends on the accuracy of the load cell (i.e., $65 \mathrm{mN}$ ). Concerning the indentation depth, the polynomial function in Eq. 4 is applied to a sensor reading affected by a given uncertainty $\Delta B_{z}$. Considering $\delta(t)$ as expressed in Eq. 3, we can write its absolute error as a function of $\Delta B_{z}$ and $\Delta d_{R}$ :

$$
|\Delta \delta| \leq\left|\frac{\partial \Phi^{-1}\left[B_{z}(t)\right]}{\partial B_{z}(t)}\right| \cdot\left|\Delta B_{z}\right|+\left|\Delta d_{R}\right| .
$$

Considering Eq. 3 and a negligible error in $d_{R}-\mathrm{a}$ reasonable assumption given the high manipulator position resolution, we then have

$$
|\Delta \delta|=\left|\sum_{i=1}^{5} i \cdot a_{i} \cdot B_{z}(t)^{i-1}\right| \cdot\left|\Delta B_{z}\right| .
$$

This equation clearly shows how the accuracy of the proposed method depends upon the strength of the magnetic field at the WPD, which, for the proposed platform, is a function of the distance between the external magnet and the WPD. Thus, higher accuracy is obtained in a stronger magnetic field (i.e., the shorter the distance between the WPD and the external magnet).

\section{EXPERIMENTAL RESULTS}

Experimental validation of single degree of freedom wireless palpation consisted in three different trials. First, the effectiveness of the algorithm in reconstructing the indentation depth from magnetic field values was assessed. Then, three silicone tissue samples, each with a different elastic modulus, were indented with the proposed approach, and the results compared with standard indentation. Finally, a lump was embedded in a porcine liver and wireless palpation was used to identify differences in tissue stiffness.

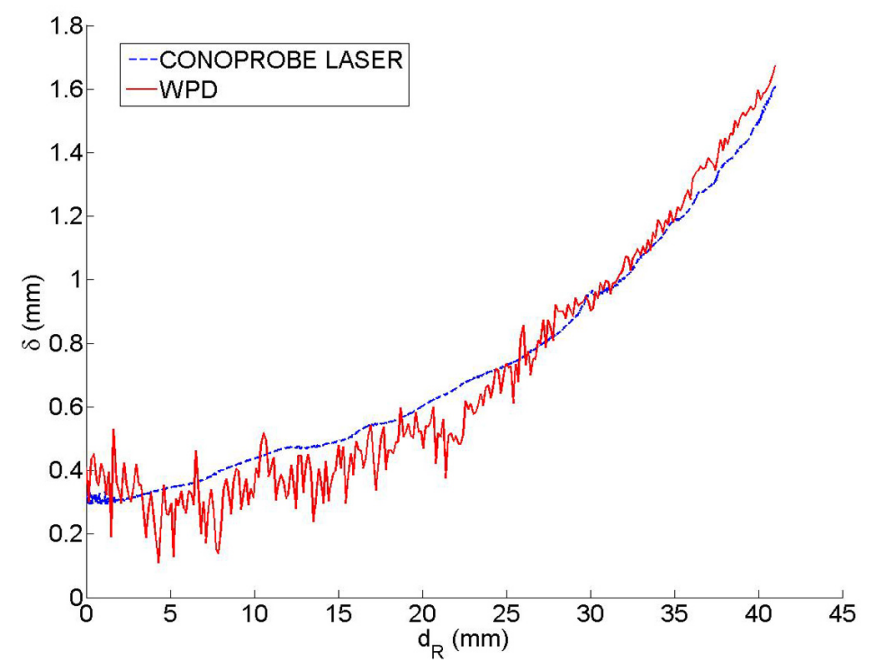

Fig. 5. Tissue indentation depth plotted as a function of $d_{R}$ and measured via the conoprobe laser and via the proposed WPD approach.

\section{A. Indentation algorithm assessment}

An optical conoscopic holography sensor (Conoprobe, Optimet, USA) was adopted as reference measurement system. The conoprobe laser was pointed at the upper circular surface of the WPD, as shown in Fig. 3. The indentation test was performed 


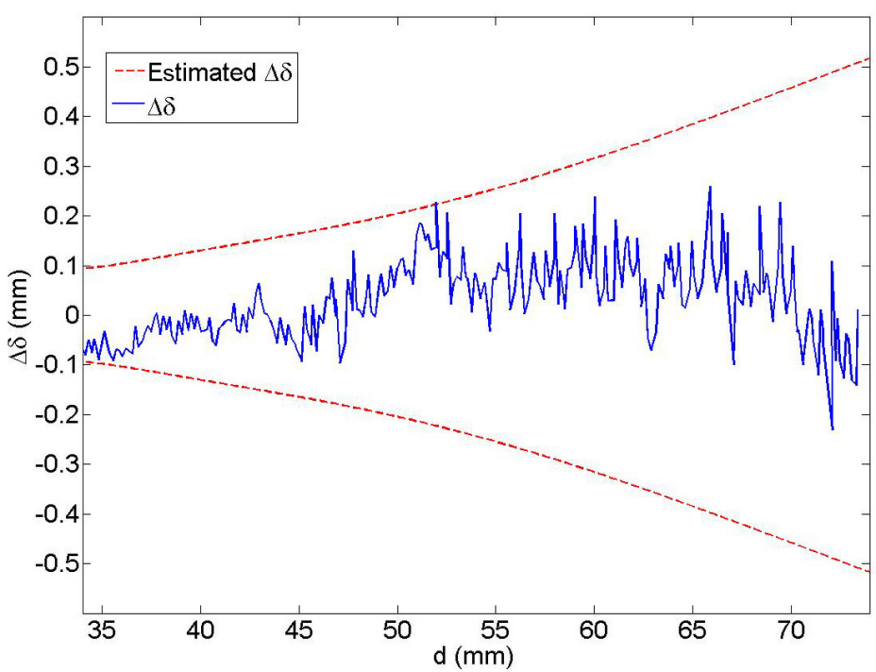

Fig. 6. Tissue indentation depth error $\Delta \delta$ as a function of $d$ for the WPD approach. The two dashed lines represent the envelope of the maximum measurement error for the tissue indentation depth, $\Delta \delta$, as a function of the distance $d$.

on a squared silicone tissue sample (elastic modulus 64.49 $\mathrm{kPa}$, thickness $35 \mathrm{~mm}$, lateral side $75 \mathrm{~mm}$ ) for $d_{R}$ varying at a constant speed (i.e., $3.12 \mathrm{~mm} / \mathrm{s}$ ) from $0 \mathrm{~mm}$ to $41 \mathrm{~mm}$, where for $d_{R}=41 \mathrm{~mm}$ the top part of the external magnet holder was almost in contact with the lower side of the rigid support. Five loading-unloading trials were completed and error analysis was performed on the acquired data. Accelerometer output confirmed that WPD motion was only occurring along the $\mathrm{Z}$ direction.

A typical loading plot for $\delta(t)$ acquired with both the reference system and the proposed approach is represented in Fig. 5 as a function of $d_{R}$. Considering the tissue sample thickness, the rigid support, and the recorded indentation depth, the distance $d$ from the external magnet to the WPD varied from $75 \mathrm{~mm}$ to $35 \mathrm{~mm}$ during the trials.

Concerning the error, the Hall effect sensor measurements presented a maximum error of $\pm 0.3 \mathrm{mT}$. By using this value in Eq. 6 as $\Delta B_{z}$, it is possible to plot an envelope of the expected maximum measurement error of the tissue indentation depth $\delta$ as a function of the distance $d$ (Fig. 6). For all of the acquired measurements, the difference between the conoprobe laser reading and the reconstructed $\delta$ always fell within the envelope. One example is given in Fig. 6. From the same plot it is possible to see that the measurement error for $\delta$ is \pm 0.1 $\mathrm{mm}$ at $35 \mathrm{~mm}$, and increasing to $\pm 0.5 \mathrm{~mm}$ at $75 \mathrm{~mm}$.

\section{B. In vitro trials}

In order to validate the effectiveness of wireless palpation to detect the elastic modulus like done when using a traditional indenter, three squared silicone tissue simulators (thickness $35 \mathrm{~mm}$, lateral side $75 \mathrm{~mm}$ ) were fabricated, each with a different proportion of hardener (i.e., 20\%, 25\%, and 30\%), thus resulting in different elastic moduli E1, E2, and E3 [25]. A traditional vertical indenter was obtained by replacing the magnet holder with a cylindrical probe at the interface with
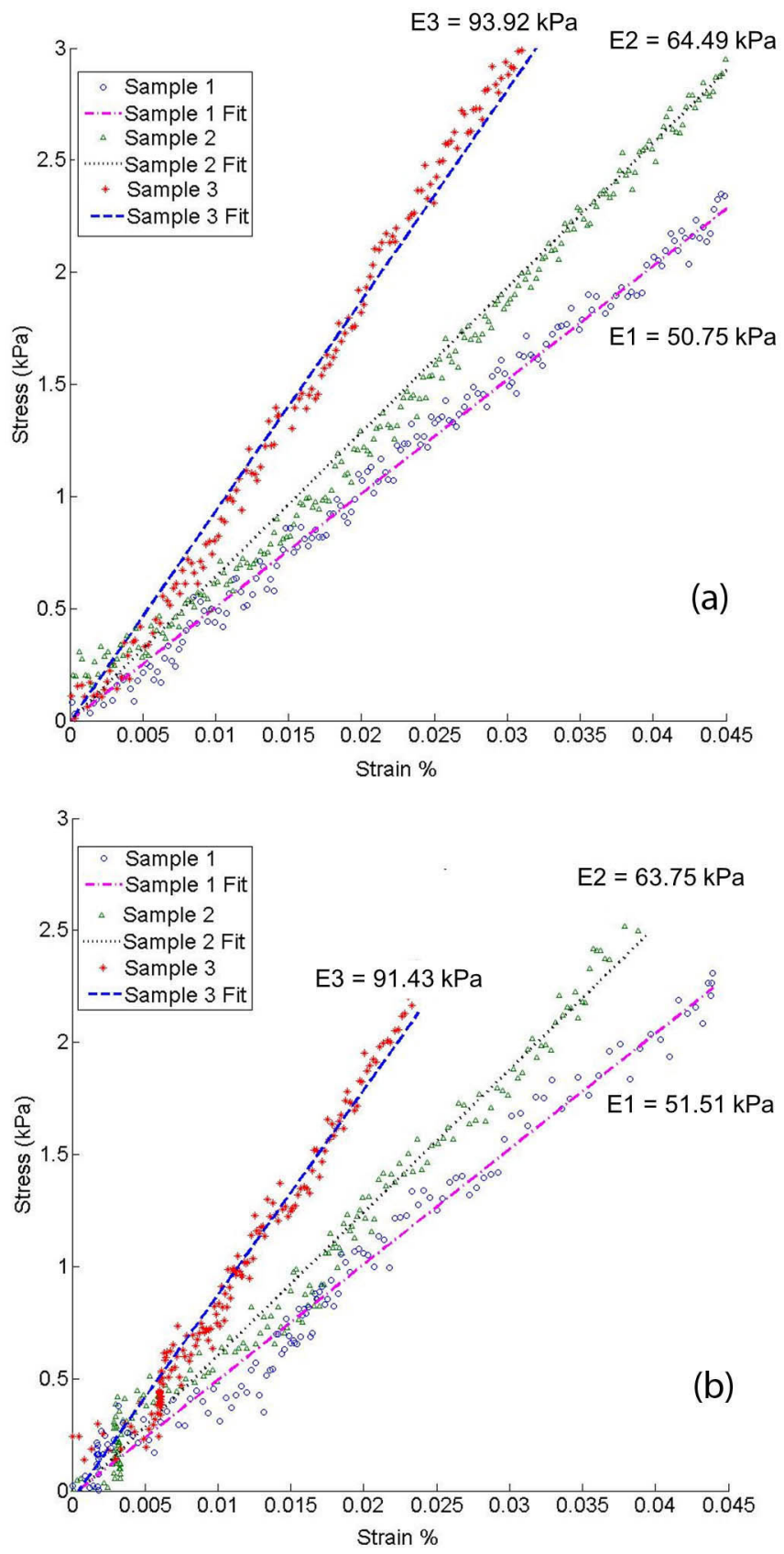

Fig. 7. Experimental data acquired by standard indentation (a) and by wireless indentation (b) for three different silicone tissue samples.

the load cell. The probe was designed to have the same contact area as the WPD. The indenter probe was first driven to touch the surface of the tissue layer with a preload of $0.2 \mathrm{~N}$. Five loading-unloading trials - reaching an indentation depth of 3 $\mathrm{mm}$ - were performed for each tissue sample at a constant speed of $3.12 \mathrm{~mm} / \mathrm{s}$. Stress-strain plots obtained from a single loading are represented in Fig.7a. Elastic moduli obtained by least square fitting were $\mathrm{E} 1=50.75 \mathrm{kPa}\left(R^{2}=0.9973\right), \mathrm{E} 2=$ $64.49 \mathrm{kPa}\left(R^{2}=0.9935\right)$, and $\mathrm{E} 3=93.92 \mathrm{kPa}\left(R^{2}=0.9972\right)$.

Wireless palpation was then performed on the same three 
samples. Five loading-unloading trials were performed by following the same protocol described for the assessment of the indentation algorithm (see Sec. III-A). The results are reported in Fig. 7b. Also in this case, accelerometer data confirmed that WPD motion was always occurring along the $\mathrm{Z}$ direction. Indentation force reached $1.26 \mathrm{~N}$, while indentation depth ranged from $0.85 \mathrm{~mm}$ for the stiffer sample to 1.57 $\mathrm{mm}$ for the softer sample. Elastic moduli obtained by least square fitting were $\mathrm{E} 1=51.51 \mathrm{kPa}\left(R^{2}=0.9825\right), \mathrm{E} 2=$ $63.75 \mathrm{kPa}\left(R^{2}=0.9802\right)$, and $\mathrm{E} 3=91.43 \mathrm{kPa}\left(R^{2}=0.9608\right)$. Considering all the performed trials, the average relative error for wireless palpation in measuring the elastic module was $1.5 \%, 1.1 \%$ and $2.8 \%$ for the tissue samples having E1, E2, and E3, respectively. The largest relative error occurred for the stiffer tissue sample. This can be explained by considering that the indentation depth for this sample was the smallest, thus the distance between the WPD and the external magnet was the largest among the trials, leading to the largest error.

\section{Ex vivo trials}

A freshly excised porcine liver (maximum length $250 \mathrm{~mm}$, maximum width $140 \mathrm{~mm}$, maximum thickness $40 \mathrm{~mm}$, weight $450 \mathrm{~g}$ ) was used for the ex vivo trials to verify whether the WPD was able to detect a stiffer area in the organ. Since in this study the motion of the WPD was not constrained to the vertical axis, but the sensing technique was limited to the vertical direction, we decided to use a squared lump instead of a spherical nodule - as suggested in [3] and [15], in order to minimize lateral motions of the WPD. The squared lump was made from hard material (Objet VeroWhite material, elastic modulus stiffer than $1100 \mathrm{MPa}$ ) using a rapid prototyping machine; the lump was $2 \mathrm{~mm}$ thick and $16 \mathrm{~mm}$ in side, so to cover a larger area than the WPD. The lump was embedded $10 \mathrm{~mm}$ beneath the tissue surface so as to simulate a hidden malignant liver tumor, usually stiffer than the surrounding healthy tissue.

Wireless indentation was performed $20 \mathrm{~mm}$ on the left from where the lump was embedded (i.e., position A in Fig. 8aWPD during palpation represented in Fig. 8b) and directly on top of the lump (i.e., position B in Fig. 8a). The same protocol described for the assessment of the indentation algorithm (see Sec. III-A) was followed and the accelerometer data confirmed that WPD motion was occurring along the $\mathrm{Z}$ direction. The results are reported in Fig. 9. Indentation force reached $3 \mathrm{~N}$, while maximum indentation depth was $3.5 \mathrm{~mm}$. Elastic moduli obtained by least square fitting at the two positions were $\mathrm{E}_{A}=$ $46.01 \mathrm{kPa}\left(R^{2}=0.9622\right)$ and $\mathrm{E}_{B}=67.50 \mathrm{kPa}\left(R^{2}=0.9669\right)$.

\section{CONClusions And Future Work}

While most research has focused either on providing force and tactile sensing at the end effector, or enabling haptic rendering at the user interface, the proposed approach tackles the physical connection between the two sides of the palpation instrument. The reported results lead to the conclusion that wireless vertical indentation is feasible in a laboratory setting, showing comparable results to traditional indentation

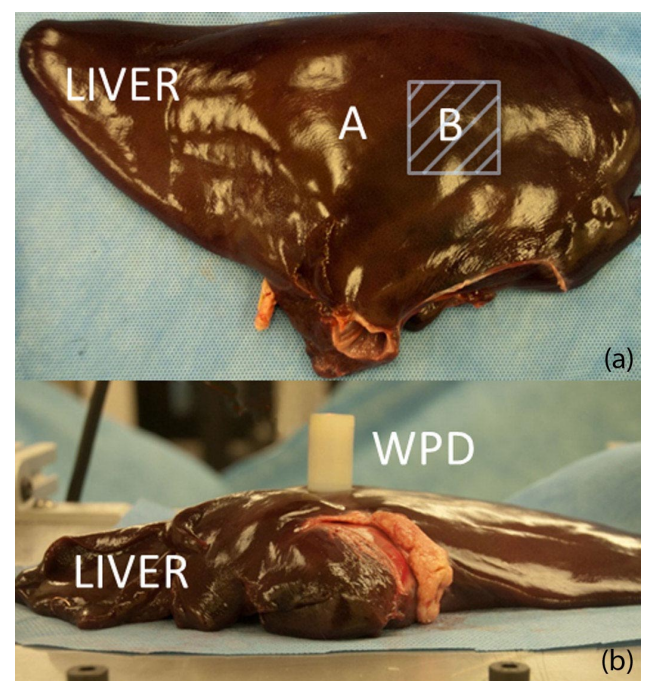

Fig. 8. Liver sample where A and B highlight the positions where wireless palpation was performed. The squared lump was embedded $10 \mathrm{~mm}$ beneath the tissue in position B, as represented by the squared overlay. Distance between position A and B is $20 \mathrm{~mm}$ (a). Side view of the porcine liver with the WPD above position A (b).

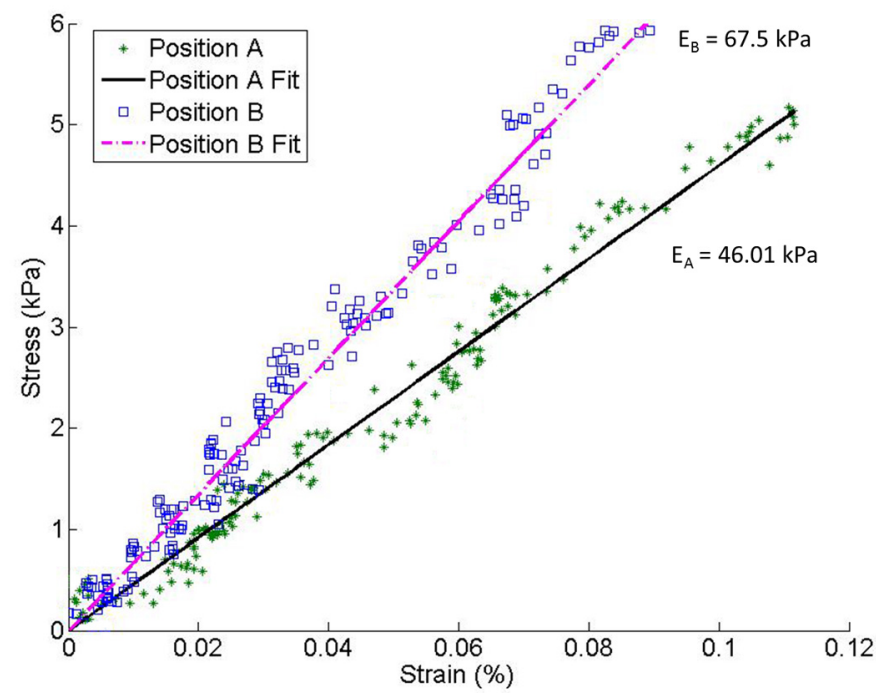

Fig. 9. Experimental data acquired by wireless vertical indentation on on the liver surface in positions $\mathrm{A}$ and $\mathrm{B}$.

techniques. Given the absence of a rigid link between the palpation probe and the external platform, vibrations and patient's respiration will likely affect the measurement when moving to in vivo operations. These artifacts can be potentially filtered out thanks to the onboard accelerometer. Undesired magnetic interaction with surgical tools can be prevented by using non-magnetic or plastic instruments.

Given these encouraging - yet preliminary - results, the next step is to demonstrate wireless palpation in a more realistic setting, where the motion of the palpation device is not limited to a single axis. This further step requires real-time pose (i.e., position and orientation) detection for the WPD [26], that can be used together with three-dimensional manipulation [27]- 
[29] to explore organ surfaces. The inertia of the external magnet will likely hamper the measurement of the intermagnetic force by the external load cell. Therefore, alternative techniques should be pursued to detect indentation pressure, such as measuring the magnetic field gradient at the WPD or integrating a tactile skin [30] on the external surface of the WPD. Rolling indentation [2] is also a possible future direction that can be addressed by adopting diametrically magnetized magnets and ad hoc tissue-device interaction models [31].

\section{ACKNOWLEDGMENT}

This work is supported by the National Science Foundation under Grant No. CNS-1239355 and by the National Center for Advancing Translational Sciences under Grant No.UL1TR000445-06.

\section{REFERENCES}

[1] Intuitive Surgical website: www.intuitivesurgical.com

[2] H. Liu, J. Li, X. Song, L. Seneviratne, and K. Althoefer, "Rolling indentation probe for tissue abnormality identification during minimally invasive surgery," IEEE Trans. Robot., vol. 27, no. 3, pp. 450-460, 2011.

[3] P. Puangmali, H. Liu, L. Seneviratne, P. Dasgupta, and K. Althoefer, "Miniature 3-axis distal force sensor for minimally invasive surgical palpation," IEEE/ASME Trans. Mechatronics, vol. 17, no. 4, pp. 646656, 2012.

[4] P. Dario and M. Bergamasco, "An advanced robot system for automated diagnostic tasks through palpation," IEEE Trans. Biomed. Eng., vol. 35, no. 2, pp. 118-126, 1988.

[5] R. Howe, W. Peine, D. Kantarinis, and J. Son, "Remote palpation technology," IEEE Eng. Med. Biol. Mag., vol. 14, no. 3, pp. 318-323, 1995.

[6] T. Ohtsuka, A. Furuse, T. Kohno, J. Nakajima, K. Yagyu, and S. Omata, "Application of a new tactile sensor to thoracoscopic surgery: Experimental and clinical study," The Annals of Thoracic Surgery, vol. 60, no. 3, pp. 610-614, 1995.

[7] M. Ottensmeyer and J. Salisbury, "In vivo data acquisition instrument for solid organ mechanical property measurement," in Medical Image Computing and Computer-Assisted Intervention - MICCAI 2001. Springer, 2001, pp. 975-982.

[8] E. Samur, M. Sedef, C. Basdogan, L. Avtan, and O. Duzgun, "A robotic indenter for minimally invasive measurement and characterization of soft tissue response," Medical Image Analysis, vol. 11, no. 4, pp. 361-373, 2007.

[9] J. Rosen, J. Brown, S. De, M. Sinanan, and B. Hannaford, "Biomechanical properties of abdominal organs in vivo and postmortem under compression loads," Journal of Biomechanical Engineering, vol. 130, no. 021020 , pp. 1-17, 2008.

[10] K. Lister, Z. Gao, and J. Desai, "Development of in vivo constitutive models for liver: Application to surgical simulation," Annals of Biomedical Engineering, vol. 39, pp. 1060-1073, 2011.

[11] O. Van der Meijden and M. Schijven, "The value of haptic feedback in conventional and robot-assisted minimal invasive surgery and virtual reality training: a current review," Surgical Endoscopy, vol. 23, pp. 1180-1190, 2009.

[12] G. Tholey and J. P. Desai, "A compact and modular laparoscopic grasper with tri-directional force measurement capability," ASME Journal of Medical Devices, vol. 2, no. 3, pp. 031 001-9, 2008.

[13] B. Kübler, U. Seibold, and G. Hirzinger, "Development of actuated and sensor integrated forceps for minimally invasive robotic surgery," The International Journal of Medical Robotics and Computer Assisted Surgery, vol. 1, no. 3, pp. 96-107, 2005.

[14] M. Stark, T. Benhidjeb, S. Gidaro, and E. Morales, "The future of telesurgery: a universal system with haptic sensation," Journal of the Turkish-German Gynecological Association, vol. 13, no. 1, pp. 74-76, 2012.

[15] J. Gwilliam, Z. Pezzementi, E. Jantho, A. Okamura, and S. Hsiao, "Human vs. robotic tactile sensing: Detecting lumps in soft tissue," in IEEE Haptics Symposium, 2010, pp. 21-28.
[16] G. McCreery, A. Trejos, M. Naish, R. Patel, and R. Malthaner, "Feasibility of locating tumours in lung via kinaesthetic feedback." The International Journal of Medical Robotics and Computer Assisted Surgery, vol. 4, no. 1, pp. 58-68, 2008.

[17] R. E. Goldman, A. Bajo, and N. Simaan, "Algorithms for autonomous exploration and estimation in compliant environments," Robotica, 2012, in press, available on-line.

[18] P. Culmer, J. Barrie, R. Hewson, M. Levesley, M. Mon-Williams, D. Jayne, and A. Neville, "Reviewing the technological challenges associated with the development of a laparoscopic palpation device," The International Journal of Medical Robotics and Computer Assisted Surgery, vol. 8, no. 2, pp. 146-159, 2012.

[19] B. Laulicht, N. J. Gidmark, A. Tripathi, and E. Mathiowitz, "Localization of magnetic pills," Proceedings of the National Academy of Sciences, vol. 108, no. 6, pp. 2252-2257, 2011.

[20] E. P. Furlani, Permanent Magnet and Electromechanical Devices. Academic Press, 2001, pp. 131-135.

[21] P. Valdastri, A. Menciassi, and P. Dario, "Transmission power requirements for novel ZigBee implants in the gastrointestinal tract," IEEE Trans. Biomed. Eng., vol. 55, no. 6, pp. 1705-1710, 2008.

[22] S. R. Platt, J. A. Hawks, and M. E. Rentschler, "Vision and task assistance using modular wireless in vivo surgical robots," IEEE Trans. Biomed. Eng., vol. 56, no. 6, pp. 1700-1710, 2009.

[23] C. Song, A. Alijani, T. Frank, G. Hanna, and A. Cuschieri, "Mechanical properties of the human abdominal wall measured in vivo during insufflation for laparoscopic surgery," Surgical Endoscopy, vol. 20, no. 6, pp. 987-990, 2006.

[24] C. Di Natali, T. Ranzani, M. Simi, A. Menciassi, and P. Valdastri, "Trans-abdominal active magnetic linkage for robotic surgery: Concept definition and model assessment," in Robotics and Automation (ICRA), IEEE International Conference on, 2012, pp. 695-700.

[25] M. Kunkel, A. Moral, R. Westphal, D. Rode, M. Rilk, and F. Wahl, "Using robotic systems in order to determine biomechanical properties of soft tissues," in Studies in Health Technology and Informatics, Proceedings of the 2nd Conference on Applied Biomechanics, vol. 133, no. 3, 2008, p. 156

[26] C. D. Natali, M. Beccani, and P. Valdastri, "Real-time pose detection for magnetic medical devices," IEEE Trans. Magn., 2013, in press, available on-line.

[27] G. Ciuti, P. Valdastri, A. Menciassi, and P. Dario, "Robotic magnetic steering and locomotion of capsule endoscope for diagnostic and surgical endoluminal procedures," Robotica, vol. 28, no. 2, pp. 199-207, 2010.

[28] S. Yim and M. Sitti, "Design and rolling locomotion of a magnetically actuated soft capsule endoscope," IEEE Trans. Robot., vol. 28, no. 1, pp. 183-194, 2012.

[29] A. W. Mahoney, D. L. Cowan, K. M. Miller, and J. J. Abbott, "Control of untethered magnetically actuated tools using a rotating permanent magnet in any position," in Robotics and Automation (ICRA), IEEE International Conference on, 2012, pp. 3375-3380.

[30] Takktile by Y. Tenzer, L. Jentoft, I. Daniher, and Robert Howe: www.takktile.com .

[31] M. E. Rentschler, J. Dumpert, S. R. Platt, K. Lagnernma, D. Oleynikov, and S. M. Farritor, "Modeling, analysis, and experimental study of in vivo wheeled robotic mobility," IEEE Trans. Robot., vol. 22, no. 2, pp. 308-321, 2006. 